\section{In a Blinded Analysis, Restriction Endonuclease Fingerprinting Detects All the Mutations in a 1.9-kb Segment}

\section{BioTechniques 23:836-839 (November 1997)}

Single-strand conformation polymorphism (SSCP) (4) is the most widely used DNA screening method. SSCP detects single-base sequence changes by abnormal electrophoretic migration of one or both single strands on a nondenaturing gel. The method does not detect all sequence changes, and the sensitivity is a complex function of sequence context and size (1). Dideoxy fingerprinting (ddF) and bi-directional dideoxy fingerprinting (bi-ddF) were developed as hybrids of SSCP and either conventional Sanger dideoxy sequencing or bidirectional cycle sequencing. DdF and bi-ddF generate substantial redundancy in the SSCP effect, thereby detecting virtually $100 \%$ of single-base changes in segments of $<300 \mathrm{bp}$ and 300-600 bp, respectively $(2,6)$.

Restriction endonuclease fingerprinting (REF) is another hybrid method, which can detect virtually all mutations in a $1-\mathrm{kb}$ segment (3). We show that twice as much sequence can be screened with REF ( $2 \mathrm{~kb}$ from 50 samples in a typical manual gel) with a small increase in effort. In addition, we provide evidence that REF is both a workhorse technique and can be applied to genes with GC contents of $70 \%$.

For REF, a DNA segment is amplified by polymerase chain reaction
(PCR), digested separately with different restriction enzymes, mixed, labeled, denatured and electrophoresed on a nondenaturing gel. Previously, five different restriction endonuclease digestions were performed in a blinded analysis that detected all of 51 mutations in a 1-kb segment (3). We use six groups of restriction endonucleases to analyze a $1.94-\mathrm{kb}$ segment of the human factor IX gene. Thus, any sequence change will be present in six restriction segments that yield twelve single-stranded segments (unless the mutation creates or destroys one or more of the restriction endonuclease sites). A change in mobility in any one of these segments is sufficient to detect the presence of a mutation. A change in mobility might reflect either a change in secondary structure (the SSCP component of REF) or an altered restriction

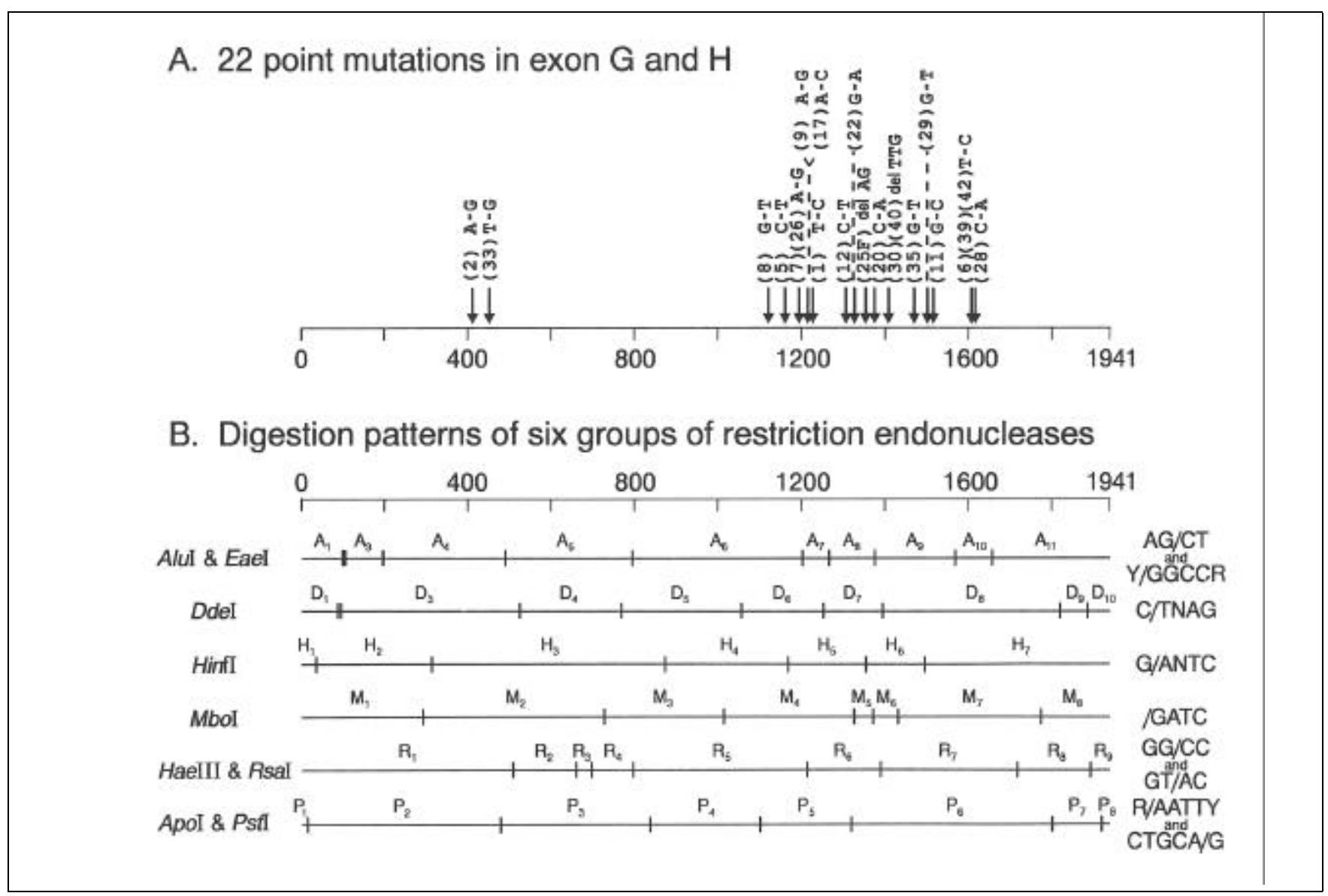

Figure 1. (A) REF detection of point mutations in exons G and H. A 1941-bp region was screened from bp 29705 to 31645 [number system by Yoshitake et al. (9)], and the bp 29705 is assigned at one above. The mutation types and positions are shown above and ordered in Figure 2, A and B. Samples 6, 39 and 42 contain the same mutation, as do samples 30 and 40. (B) Digestion pattern of 6 groups of restriction endonucleases. Restriction maps are shown above for each of the 6 groups of restriction endonucleases: AluI and EaeI (A), DdeI (D), HinfI (H), MboI (M), HaeIII and RsaI (R), ApoI and PstI (P). The digested segments are numbered according to the enzyme used and the linear order of the segments. ApoI with optimal incubation temperature of $50^{\circ} \mathrm{C}$ was incubated at $37^{\circ} \mathrm{C}(50 \%$ activity) together with PstI. 
Table 1. Summary of Analysis of 19 Different Point Mutationsa

\begin{tabular}{|lcc|}
\hline Measure & At $\mathbf{2 0}^{\circ} \mathbf{C}(\%)$ & At $\mathbf{8}^{\circ} \mathbf{C}(\%)$ \\
\hline $\begin{array}{l}\text { Sensitivity } \\
\text { (restriction component)a }\end{array}$ & 22.2 & 22.2 \\
$\begin{array}{l}\text { Average Efficiency } \\
\text { (SSCP component)b,c }\end{array}$ & 65.6 & 76.3 \\
Sensitivity (SSCP)b & & \\
Sensitivity (REF) & 100 & 100 \\
& 100 & 100
\end{tabular}

aFour single-base mutations (lanes 9, 17, 20 and 22) were associated with loss of restriction sites (two lost $R$ sal sites and two lost $M b o l$ sites). These informative restriction components were detected easily.

bThe average SSCP component efficiency is determined by dividing the number of informative segments for all the mutations by all the segments in the SSCP component. The efficiency of the SSCP component for a given mutation is the number of informative segments divided by the total number of segments in the SSCP component. The sensitivity of the SSCP component is the percentage of samples in which the presence of a mutation could be detected; i.e., the percentage in which the efficiency of the SSCP component is greater than zero.

cThe total number of scorable mutation-containing segments in the SSCP components was 224 at $20^{\circ} \mathrm{C}$ and 178 at $8^{\circ} \mathrm{C}$, since some segments were electrophoresed out of the gel, dispersed or co-migrated. The segments of the SSCP component were scored by visual analysis for the presence of abnormal migration, and unequivocal mobility changes were scored. Typically, a migration change of $1 / 2$ bandwidth was taken as the resolution. Also, a wild-type band may be composed of two or more compressed segments, partially or completely. If a mutation resolved into separate bands or if a mutation compressed separate segments (e.g., lane 26 in Figure 2B), the segments were scored as abnormal. Mutant No. 8 showed the lowest efficiency (16.6\%), while the efficiencies of 7 different mutants were $90+\%$.

site (the restriction component of REF).

A 1.94-kb segment was amplified with the following primers: [informative name: I6(29705)-28D; 5'-CCC ATG AAG CAA ATT TTG CCA TCA GAG A-3'] and B [E8(31645)-31U 5'CTG ATG GAC AGA GTG GAG AAT TTA ACT TCA C-3'] [number system from Reference 8; informative primer names as described in Sarkar et al. (5)]. The restriction endonucleases were selected by visual examination of the restriction map (Figure 1). PCR-amplified DNA (100 ng) was digested in separate tubes with six groups of restriction endonucleases as follows: (i) 5 $\mathrm{U}$ of $A l u \mathrm{I}$ and $1 \mathrm{U}$ of EaeI in Reaction Buffer 1; (ii) $0.75 \mathrm{U}$ of DdeI in Buffer 3; (iii) $4 \mathrm{U}$ of HinfI in Buffer 2; (iv) $1.25 \mathrm{U}$ of $\mathrm{MboI}$ in Buffer 3; (v) $2.5 \mathrm{U}$ of HaeIII and $5 \mathrm{U}$ of RsaI in Buffer 2; or (vi) $2 \mathrm{U}$ of ApoI and $6 \mathrm{U}$ of Pst $\mathrm{I}$ in Buffer 3 (unless mentioned specifically, all enzymes and buffers are from New England Biolabs [Beverly, MA, USA]). Each digestion produced 7-10 restric- tion segments, resulting in a ladder of 104 single-stranded DNA segments per lane $(8.6$ segments per digestion $\times 6$ digestions $\times 2$ strands). The average size of the digested segments was $223 \mathrm{bp}$. The above reaction mixtures contained $0.5 \mathrm{U}$ of calf intestinal alkaline phosphatase (CIAP) to remove the phosphate group at the $5^{\prime}$ end of the digested DNA segments. A final volume of 10 $\mu \mathrm{L}$ was incubated at $37^{\circ} \mathrm{C}$ for $6 \mathrm{~h}$, and then the enzymes were inactivated by heating at $80^{\circ} \mathrm{C}$ for $30 \mathrm{~min}$. The digestions were combined (yielding $60 \mu \mathrm{L}$ ), reheated at $90^{\circ} \mathrm{C}$ for 3 min and quickchilled. To facilitate equal labeling of the single-stranded ends, $3 \mathrm{ng}(0.33$ $\mu \mathrm{L})$ of digested DNA products were $5^{\prime}$ end-labeled with $5 \mu \mathrm{Ci}\left[\gamma_{-32}{ }^{32}\right]$ ATP and $1 \mathrm{U}$ of T4 polynucleotide kinase. The reaction volume was $2 \mu \mathrm{L}$, and it was incubated at $37^{\circ} \mathrm{C}$ for $45 \mathrm{~min}$. Stop/ loading buffer $(50 \mu \mathrm{L} ; 7 \mathrm{M}$ urea, $50 \%$ formamide, $2 \mathrm{mM}$ EDTA) was added to each sample. Each sample $(1.5 \mu \mathrm{L})$ was electrophoresed for 4-6 h through a 


\section{Benchmarks}

7.5\% GeneAmp ${ }^{\circledR}$ gel (Perkin-Elmer, Norwalk, CT, USA) with TBE buffer (50 mM Tris-borate and $1 \mathrm{mM}$ EDTA, $\mathrm{pH} 8.2$ ) at $20^{\circ}$ or at $8^{\circ} \mathrm{C}$.

The above protocol differs from the previous protocol (3) in the following ways: $(i)$ six restriction endonucleases, rather than five, were used; (ii) $0.5 \mathrm{U}$ of CIAP was used regardless of whether the restriction endonucleases generated 5 ' overhangs, blunt ends or 3' overhangs; (iii) inactivation of the restriction endonucleases and CIAP was performed at $80^{\circ} \mathrm{C}$ (rather than $94^{\circ} \mathrm{C}$ ) for $30 \mathrm{~min}$, thereby eliminating the background associated with the gels; (iv) 104 single-stranded segments were analyzed per lane, as compared to 58 single-stranded segments previously; and $(v)$ the segments were significantly larger on average (an average of about
$224 \mathrm{bp}$ with the largest segment of 559 bp compared to the previous experiment, in which the average segment was about $150 \mathrm{bp}$, and the largest segment was $285 \mathrm{bp}$ ).

In this study, forty-two samples of DNA from Hemophilia B patients were tested blindly in exons $\mathrm{G}$ and $\mathrm{H}$. Twenty-two of the mutant samples contained a total of 18 different mutations (Figure 1A). A 1941-bp PCR segment was amplified and then cleaved separately by six groups of restriction endonucleases. All 18 mutations $[15$ single-base substitutions and two microdeletions of 2 and 3 bp (Figure 1A)] were found by REF and confirmed by sequencing analysis (Figures 1 and 2). There were no false-positives, and the repeated mutations gave reproducible fingerprints (Figure 2, lanes 6, 39 and 42; lanes 30 and 40). Thus, the sensitivity of REF was extended from an average segment size (about $150 \mathrm{bp}$ ) used in the previous protocol to about $220 \mathrm{bp}$.

A mutation can be localized by the restriction and/or SSCP components. Abnormal segments were scored and localized by comparison with control segments produced by digestion with a single group of restriction endonucleases (6 right-most lanes). Thirteen of the eighteen independent mutations could be localized within a 100-bp region, and all the mutations were localized within a 282-bp region. These results reflect the high efficiency of the SSCP component of REF (Table 1).

REF has been a workhorse technique in the laboratory. It has been applied to multiple exonic and intronic regions of the factor IX gene, the

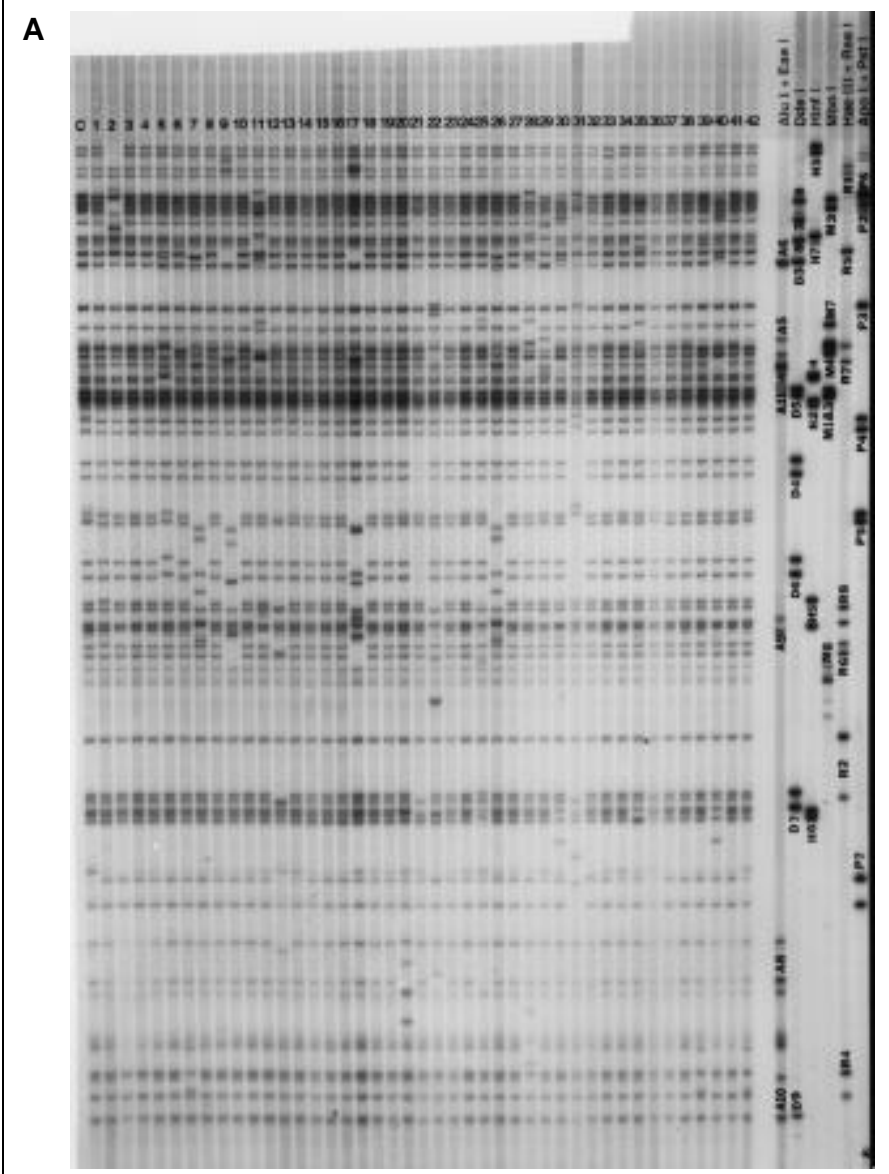

\section{B}

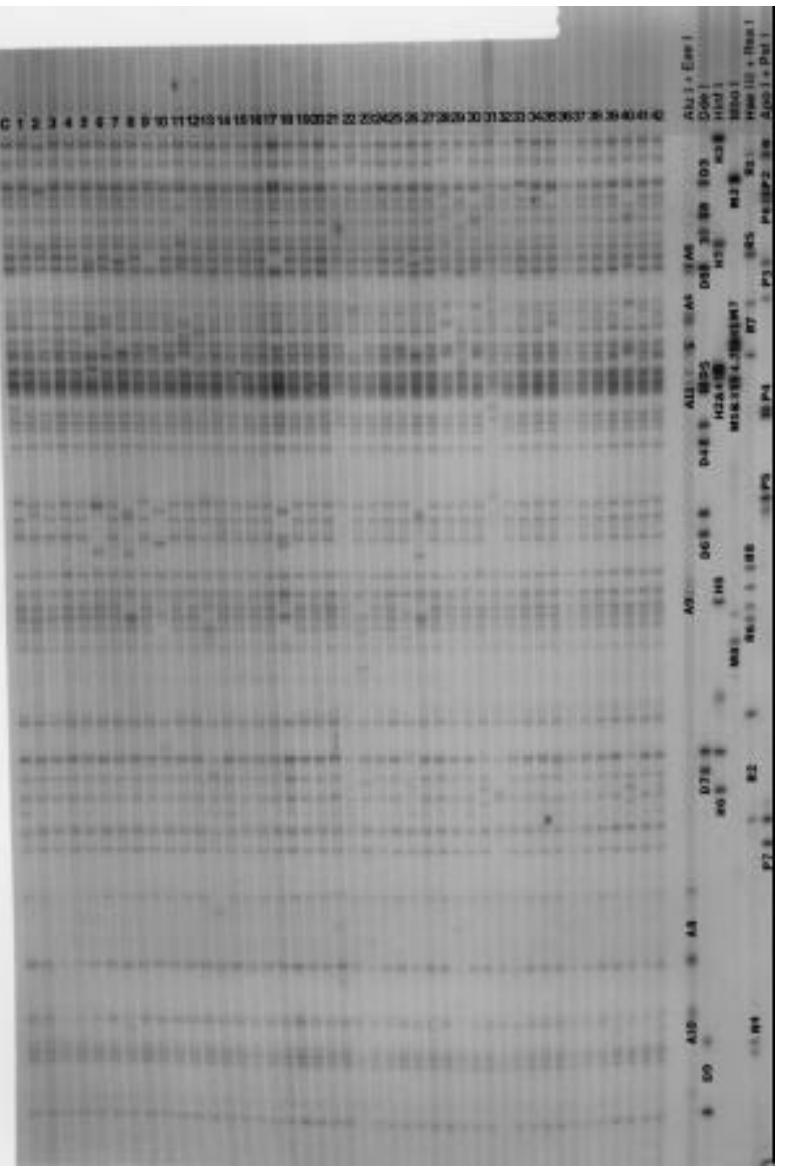

Figure 2. REF autoradiography for screening unknown DNA samples in a 1941-bp region. (A) Nondenaturing gel electrophoresis at $20^{\circ} \mathrm{C}$. Lane C: wildtype sequence control. Lanes 1-42: unknown DNA samples. On the right side of the gel are digestion products from each enzyme group. These control reactions serve to identify the segments in an REF fingerprint. Segments of A7 and M5 were electrophoresed out of the gel. The lanes with mutations are shown in Figure 1A. (B) Nondenaturing gel electrophoresis at $8^{\circ} \mathrm{C}$. Segments of A7, M5 and M6 were electrophoresed out of the gel, and one strand of the A9 segment was dispersed; i.e., smeared. 
dopamine D1 and D5 receptor genes and the $\alpha 2$ adrenergic receptor gene (data not shown). To optimize sensitivity of mutation detection near the ends of the amplified segments, a 7-8-nucleotide sequence with high GC content (e.g., GCCTGGT) and sequences containing appropriate restriction endonuclease sites were incorporated into the $5^{\prime}$ end of the PCR primers. The high $\mathrm{GC}$ sequence is lost after digestion with some of the restriction endonucleases, thereby preventing all the end fragments from being co-terminal. With a standard manual gel, we typically screen fifty samples of about $2 \mathrm{~kb}$ for a total of about $100 \mathrm{~kb}$ of DNA sequence for the factor IX gene in hemizygous males and $200 \mathrm{~kb}$ for the autosomal neurotransmitter receptors. One of the authors (J.F.) has utilized REF to screen a total of $3.1 \mathrm{Mb}$ of human genomic DNA with part-time effort during the past two years and discovered 52 sequence variants. In addition, other laboratories have used REF to analyze the L1 cell adhesion molecule (LICAM) gene and the ataxia telangiectasia gene (7). For regions of high GC content, it is necessary to add urea to keep the bands from smearing. Based on the above experience, we propose that thin bands on the gel can be preserved while maintaining high sensitivity if the following urea concentrations are used with electrophoresis at $20^{\circ} \mathrm{C}: 0.5 \mathrm{M}$ with a GC content of $60 \%$; $1 \mathrm{M}$ with a GC content of $65 \%$; and $1.5 \mathrm{M}$ with a GC content of $70 \%$.

REF is a highly sensitive method of screening for the presence of mutations as long as a sufficient number of restriction endonuclease digestions is performed. REF Select software has been designed to help the user choose the restriction endonuclease groups appropriate for a given sequence (Scaringe and Sommer, unpublished). The main disadvantage of REF is that multiple steps are required, thereby increasing the opportunity for technical error. For segments of less than $300 \mathrm{bp}$ or 300-600 bp, ddF and bi-ddF are more rapid methods of screening for mutations. However, for regions of $1-2 \mathrm{~kb}$, REF offers the opportunity to screen megabases of DNA in a short period of time. Efforts are underway to increase the range of REF to 3-4-kb segments.
In conclusion, the present paper extends previous work by: (i) doubling the range in which virtually all mutations are detected, as demonstrated by a blinded analysis; (ii) modifying the protocol to facilitate the method and reduce background on the fingerprint; and (iii) demonstrating that REF can be performed on genes with GC contents ranging from $50 \%-70 \%$ with the presence of appropriate concentrations of urea (52 sequence variants found in 16 analyzed regions).

\section{REFERENCES}

1.Cotton, R.G.H. 1997. Mutation Detection, p. 32-58. Oxford University Press, New York.

2.Liu, Q. and S.S. Sommer. 1994. Parameters affecting the sensitivities of dideoxy fingerprinting and SSCP. PCR Methods Appl. 4:97108.

3.Liu, Q. and S.S. Sommer. 1995. Restriction endonuclease fingerprinting (REF): a sensitive method for screening mutations in long, contiguous segments of DNA. BioTechniques 18:470-477.

4.Orita, M., H. Iwahana, H. Kanazawa, K. Hayashi and T. Sekiya. 1989. Detection of polymorphisms of human DNA by gel electrophoresis as single-strand conformation polymorphisms. Proc. Natl. Acad. Sci. USA 86:2766-2770.

5.Sarkar, G., S. Kapelner and S.S. Sommer. 1990. Formamide can dramatically improve the specificity of PCR. Nucleic Acids Res. 18:7465.

6.Sarkar, G., H. Yoon and S.S. Sommer. 1992. Dideoxy fingerprinting (ddF): a rapid and efficient screen for the presence of mutations. Genomics 13:441-443.

7.Savitsky, K., A. Bar-Shira, S. Gilad, G. Rotman, Y. Ziv, L. Vanagaite, D.A. Tagle, S. Smith et al. 1995. A single ataxia telangiectasia gene with a product similar to P1-3 kinase. Science 268:1749-1753.

8.Yoshitake, S., B.G. Schach, D.C. Foster, E.W. Davie and K. Kurachi. 1985. Nucleotide sequence of the gene for human factor IX (anti-hemophilic factor B). Biochemistry $24: 3736-3750$.

Address correspondence to Steve $S$. Sommer, Departments of Molecular Diagnosis and Molecular Genetics, City of Hope, Duarte, CA 91010, USA.

Received 4 March 1997; accepted 9 July 1997.
Qiang Liu, Jinong Feng and Steve S. Sommer Mayo Clinic/Foundation Rochester, MN, USA 